\title{
Open Problems for Painlevé Equations
}

\author{
Peter A. CLARKSON
}

School of Mathematics, Statistics and Actuarial Science, University of Kent, Canterbury, Kent, CT2 7FS, UK

E-mail: P.A.Clarkson@kent.ac.uk

URL: https://www.kent.ac.uk/smsas/our-people/profiles/clarkson_peter.html

Received January 18, 2019; Published online January 29, 2019

https://doi.org/10.3842/SIGMA.2019.006

\begin{abstract}
In this paper some open problems for Painlevé equations are discussed. In particular the following open problems are described: (i) the Painlevé equivalence problem; (ii) notation for solutions of the Painlevé equations; (iii) numerical solution of Painlevé equations; and (iv) the classification of properties of Painlevé equations.
\end{abstract}

Key words: Painlevé equations; open problems

2010 Mathematics Subject Classification: 33E17; 34M55

\section{Introduction}

The Painlevé equations are now regarded as "nonlinear special functions", being nonlinear analogs of the classical special functions and form the core of "modern special function theory" [44, 68, 76, 138]. Indeed Iwasaki, Kimura, Shimomura and Yoshida [83] characterize the Painlevé equations as "the most important nonlinear ordinary differential equations" and state that "many specialists believe that during the twenty-first century the Painlevé functions will become new members of the community of special functions". Subsequently this has happened as the Painlevé equations are a chapter in the NIST Digital Library of Mathematical Functions [118, Section 32]. The Painlevé functions have greatly expanded the role that the classical special functions, such as the Airy, Bessel, Hermite, Legendre and hypergeometric functions, started to play in the 19th century. Increasingly, as nonlinear science develops, people are finding that the solutions to an extraordinarily broad array of scientific problems, from neutron scattering theory, special solutions of partial differential equations such as nonlinear wave equations, fibre optics, transportation problems, combinatorics, random matrices, quantum gravity and to number theory, can be expressed in terms of solutions of the Painlevé equations.

The Painlevé equations $\left(\mathrm{P}_{\mathrm{I}}-\mathrm{P}_{\mathrm{VI}}\right)$, whose solutions are called the Painlevé transcendents, are the nonlinear ordinary differential equations given by

$$
\begin{aligned}
\frac{\mathrm{d}^{2} w}{\mathrm{~d} z^{2}} & =6 w^{2}+z \\
\frac{\mathrm{d}^{2} w}{\mathrm{~d} z^{2}} & =2 w^{3}+z w+\alpha \\
\frac{\mathrm{d}^{2} w}{\mathrm{~d} z^{2}} & =\frac{1}{w}\left(\frac{\mathrm{d} w}{\mathrm{~d} z}\right)^{2}-\frac{1}{z} \frac{\mathrm{d} w}{\mathrm{~d} z}+\frac{\alpha w^{2}+\beta}{z}+\gamma w^{3}+\frac{\delta}{w}, \\
\frac{\mathrm{d}^{2} w}{\mathrm{~d} z^{2}} & =\frac{1}{2 w}\left(\frac{\mathrm{d} w}{\mathrm{~d} z}\right)^{2}+\frac{3}{2} w^{3}+4 z w^{2}+2\left(z^{2}-\alpha\right) w+\frac{\beta}{w},
\end{aligned}
$$

This paper is a contribution to the Special Issue on Orthogonal Polynomials, Special Functions and Applications (OPSFA14). The full collection is available at https://www.emis.de/journals/SIGMA/OPSFA2017.html 


$$
\begin{aligned}
\frac{\mathrm{d}^{2} w}{\mathrm{~d} z^{2}}= & \left(\frac{1}{2 w}+\frac{1}{w-1}\right)\left(\frac{\mathrm{d} w}{\mathrm{~d} z}\right)^{2}-\frac{1}{z} \frac{\mathrm{d} w}{\mathrm{~d} z}+\frac{(w-1)^{2}}{z^{2}}\left(\alpha w+\frac{\beta}{w}\right)+\frac{\gamma w}{z}+\frac{\delta w(w+1)}{w-1}, \\
\frac{\mathrm{d}^{2} w}{\mathrm{~d} z^{2}}= & \frac{1}{2}\left(\frac{1}{w}+\frac{1}{w-1}+\frac{1}{w-z}\right)\left(\frac{\mathrm{d} w}{\mathrm{~d} z}\right)^{2}-\left(\frac{1}{z}+\frac{1}{z-1}+\frac{1}{w-z}\right) \frac{\mathrm{d} w}{\mathrm{~d} z} \\
& +\frac{w(w-1)(w-z)}{z^{2}(z-1)^{2}}\left\{\alpha+\frac{\beta z}{w^{2}}+\frac{\gamma(z-1)}{(w-1)^{2}}+\frac{\delta z(z-1)}{(w-z)^{2}}\right\}
\end{aligned}
$$

where $\alpha, \beta, \gamma$ and $\delta$ are arbitrary constants. These six equations have attracted much attention for mathematicians and physicists during the past 40 years or so, though they were discovered by Painlevé, Gambier et al. in the late 19th and early 20th centuries, in an investigation of which second-order ordinary differential equations of the form

$$
\frac{\mathrm{d}^{2} w}{\mathrm{~d} z^{2}}=F\left(w, \frac{\mathrm{d} w}{\mathrm{~d} z}, z\right)
$$

where $F$ is rational in $w$ and $\mathrm{d} w / \mathrm{d} z$ and locally analytic in $z$, having the property that their solutions have no movable branch points. They showed that there were fifty canonical equations of the form (1.7) with this property, now known as the Painlevé property, up to a Möbius (bilinear rational) transformation

$$
W(\zeta)=\frac{a(z) w+b(z)}{c(z) w+d(z)}, \quad \zeta=\phi(z),
$$

where $a(z), b(z), c(z), d(z)$ and $\phi(z)$ are locally analytic functions. Further Painlevé, Gambier et al. showed that of these fifty equations, forty-four can be reduced to linear equations, solved in terms of elliptic functions, or are reducible to one of six new nonlinear ordinary differential equations that define new transcendental functions, see Ince [81, Chapter 14].

Following Sakai [128] and Ohyama et al. [113] (see also [114]), $\mathrm{P}_{\mathrm{III}}$ (1.3) can be classified into four cases:

(i) if $\gamma \delta \neq 0$, which is known as $\mathrm{P}_{\mathrm{III}}^{(6)}$, then set $\gamma=1$ and $\delta=-1$, without loss of generality, by rescaling $w$ and $z$ if necessary

$$
\frac{\mathrm{d}^{2} w}{\mathrm{~d} z^{2}}=\frac{1}{w}\left(\frac{\mathrm{d} w}{\mathrm{~d} z}\right)^{2}-\frac{1}{z} \frac{\mathrm{d} w}{\mathrm{~d} z}+\frac{\alpha w^{2}}{z}+\frac{\beta}{z}+w^{3}-\frac{1}{w} ;
$$

(ii) if $\gamma=0$ and $\alpha \delta \neq 0$ (or equivalently $\delta=0$ and $\beta \gamma \neq 0$ ), which is known as $\mathrm{P}_{\mathrm{III}}^{(7)}$, then set $\alpha=1$ and $\delta=-1$, without loss of generality

$$
\frac{\mathrm{d}^{2} w}{\mathrm{~d} z^{2}}=\frac{1}{w}\left(\frac{\mathrm{d} w}{\mathrm{~d} z}\right)^{2}-\frac{1}{z} \frac{\mathrm{d} w}{\mathrm{~d} z}+\frac{w^{2}}{z}+\frac{\beta}{z}-\frac{1}{w}
$$

or if $\delta=0$ and $\beta \gamma \neq 0$ set $\beta=-1$ and $\gamma=1$

$$
\frac{\mathrm{d}^{2} w}{\mathrm{~d} z^{2}}=\frac{1}{w}\left(\frac{\mathrm{d} w}{\mathrm{~d} z}\right)^{2}-\frac{1}{z} \frac{\mathrm{d} w}{\mathrm{~d} z}+\frac{\alpha w^{2}}{z}-\frac{1}{z}+w^{3}
$$

(iii) if $\gamma=\delta=0$ and $\alpha \beta \neq 0$, which is known as $\mathrm{P}_{\mathrm{III}}^{(8)}$, then set $\alpha=1$ and $\beta=-1$, without loss of generality

$$
\frac{\mathrm{d}^{2} w}{\mathrm{~d} z^{2}}=\frac{1}{w}\left(\frac{\mathrm{d} w}{\mathrm{~d} z}\right)^{2}-\frac{1}{z} \frac{\mathrm{d} w}{\mathrm{~d} z}+\frac{w^{2}}{z}-\frac{1}{z}
$$


(iv) if $\alpha=\gamma=0$ (or equivalently $\beta=\delta=0$ ) then the equation can be solved by quadratures and has no transcendental solutions.

In the sequel, we shall refer to equation (1.9) as $\mathrm{P}_{\mathrm{III}}$ rather than $\mathrm{P}_{\text {III }}^{(6)}$ since this is the generic case. Equation (1.10) is also known as the degenerate $\mathrm{P}_{\mathrm{III}}$, cf. [94, 95]. These different types of $\mathrm{P}_{\text {III }}$ were noted by Painlevé [123].

Similarly, $\mathrm{P}_{\mathrm{V}}(1.5)$ can be classified into three cases:

(i) if $\delta \neq 0$, then set $\delta=-\frac{1}{2}$, without loss of generality;

(ii) if $\delta=0$ and $\gamma \neq 0$, then the equation is known as degenerate $\mathrm{P}_{\mathrm{V}}\left(\operatorname{deg}-\mathrm{P}_{\mathrm{V}}\right)$

$$
\frac{\mathrm{d}^{2} w}{\mathrm{~d} z^{2}}=\left(\frac{1}{2 w}+\frac{1}{w-1}\right)\left(\frac{\mathrm{d} w}{\mathrm{~d} z}\right)^{2}-\frac{1}{z} \frac{\mathrm{d} w}{\mathrm{~d} z}+\frac{(w-1)^{2}}{z^{2}}\left(\alpha w+\frac{\beta}{w}\right)+\frac{\gamma w}{z},
$$

which is equivalent to $\mathrm{P}_{\mathrm{III}}$ (1.9), cf. [67, Theorem 4.2], [76, Section 34];

(iii) if $\gamma=0$ and $\delta=0$ then the equation can be solved by quadratures and has no transcendental solutions.

Each of the Painlevé equations can be written as a Hamiltonian system

$$
\frac{\mathrm{d} q}{\mathrm{~d} z}=\frac{\partial \mathcal{H}_{\mathrm{J}}}{\partial p}, \quad \frac{\mathrm{d} p}{\mathrm{~d} z}=-\frac{\partial \mathcal{H}_{\mathrm{J}}}{\partial q},
$$

for a suitable Hamiltonian function $\mathcal{H}_{\mathrm{J}}(q, p, z)[84,115,116]$. The function $\sigma(z) \equiv \mathcal{H}_{\mathrm{J}}(q, p, z)$ satisfies a second-order, second-degree ordinary differential equation, known as the "Painlevé $\sigma$-equation", whose solution is expressible in terms of the solution of the associated Painlevé equation $[84,116]$. The Painlevé $\sigma$-equations $\left(\mathrm{S}_{\mathrm{I}}-\mathrm{S}_{\mathrm{VI}}\right)$ associated with $\mathrm{P}_{\mathrm{I}}-\mathrm{P}_{\mathrm{VI}}$ respectively are

$$
\begin{aligned}
& \left(\frac{\mathrm{d}^{2} \sigma}{\mathrm{d} z^{2}}\right)^{2}+4\left(\frac{\mathrm{d} \sigma}{\mathrm{d} z}\right)^{3}+2 z \frac{\mathrm{d} \sigma}{\mathrm{d} z}-2 \sigma=0 \\
& \left(\frac{\mathrm{d}^{2} \sigma}{\mathrm{d} z^{2}}\right)^{2}+4\left(\frac{\mathrm{d} \sigma}{\mathrm{d} z}\right)^{3}+2 \frac{\mathrm{d} \sigma}{\mathrm{d} z}\left(z \frac{\mathrm{d} \sigma}{\mathrm{d} z}-\sigma\right)=\frac{1}{4} \beta^{2} \\
& \left(z \frac{\mathrm{d}^{2} \sigma}{\mathrm{d} z^{2}}-\frac{\mathrm{d} \sigma}{\mathrm{d} z}\right)^{2}+\left[4\left(\frac{\mathrm{d} \sigma}{\mathrm{d} z}\right)^{2}-z^{2}\right]\left(z \frac{\mathrm{d} \sigma}{\mathrm{d} z}-2 \sigma\right)+4 z \vartheta_{\infty} \frac{\mathrm{d} \sigma}{\mathrm{d} z}=2 \vartheta_{0} z^{2} \\
& \left(\frac{\mathrm{d}^{2} \sigma}{\mathrm{d} z^{2}}\right)^{2}-4\left(z \frac{\mathrm{d} \sigma}{\mathrm{d} z}-\sigma\right)^{2}+4 \frac{\mathrm{d} \sigma}{\mathrm{d} z}\left(\frac{\mathrm{d} \sigma}{\mathrm{d} z}+2 \vartheta_{0}\right)\left(\frac{\mathrm{d} \sigma}{\mathrm{d} z}+2 \vartheta_{\infty}\right)=0 \\
& \left(z \frac{\mathrm{d}^{2} \sigma}{\mathrm{d} z^{2}}\right)^{2}-\left[2\left(\frac{\mathrm{d} \sigma}{\mathrm{d} z}\right)^{2}-z \frac{\mathrm{d} \sigma}{\mathrm{d} z}+\sigma\right]^{2}+4 \prod_{j=1}^{4}\left(\frac{\mathrm{d} \sigma}{\mathrm{d} z}+\kappa_{j}\right)=0, \\
& \frac{\mathrm{d} \sigma}{\mathrm{d} z}\left[z(z-1) \frac{\mathrm{d}^{2} \sigma}{\mathrm{d} z^{2}}\right]^{2}+\left[\frac{\mathrm{d} \sigma}{\mathrm{d} z}\left\{2 \sigma-(2 z-1) \frac{\mathrm{d} \sigma}{\mathrm{d} z}\right\}+\kappa_{1} \kappa_{2} \kappa_{3} \kappa_{4}\right]^{2}=\prod_{j=1}^{4}\left(\frac{\mathrm{d} \sigma}{\mathrm{d} z}+\kappa_{j}^{2}\right),
\end{aligned}
$$

where $\beta, \vartheta_{0}, \vartheta_{\infty}$ and $\kappa_{1}, \ldots, \kappa_{4}$ are arbitrary constants.

Example 1.1. The Hamiltonian associated with $\mathrm{P}_{\mathrm{II}}(1.2)$ is

$$
\mathrm{H}_{\mathrm{II}}(q, p, z ; \alpha)=\frac{1}{2} p^{2}-\left(q^{2}+\frac{1}{2} z\right) p-\left(\alpha+\frac{1}{2}\right) q
$$

and so

$$
\frac{\mathrm{d} q}{\mathrm{~d} z}=p-q^{2}-\frac{1}{2} z, \quad \frac{\mathrm{d} p}{\mathrm{~d} z}=2 q p+\alpha+\frac{1}{2},
$$


see $[84,117]$. Eliminating $p$ in $(1.20)$ then $q$ satisfies $\mathrm{P}_{\text {II }}(1.2)$ whilst eliminating $q$ yields

$$
\frac{\mathrm{d}^{2} p}{\mathrm{~d} z^{2}}=\frac{1}{2 p}\left(\frac{\mathrm{d} p}{\mathrm{~d} z}\right)^{2}+2 p^{2}-z p-\frac{\left(\alpha+\frac{1}{2}\right)^{2}}{2 p},
$$

which is known as $\mathrm{P}_{34}$ since is equivalent to equation XXXIV of Chapter 14 in [81]. Hence if $q$ satisfies $\mathrm{P}_{\mathrm{II}}$ (1.2) then $p=q^{\prime}+q^{2}+\frac{1}{2} z$ satisfies (1.21). Conversely if $p$ satisfies (1.21) then $q=\left(p^{\prime}-\alpha-\frac{1}{2}\right) /(2 p)$ satisfies $\mathrm{P}_{\mathrm{II}}(1.2)$. Thus there is a one-to-one correspondence between solutions of $\mathrm{P}_{\mathrm{II}}(1.2)$ and those of $\mathrm{P}_{34}$ (1.21). Further, the function $\sigma(z ; \alpha)=\mathrm{H}_{\mathrm{II}}(q, p, z ; \alpha)$ defined by (1.19), where $q$ and $p$ satisfy the system (1.20), then $\sigma(z ; \alpha)$ satisfies (1.14). Conversely if $\sigma(z ; \alpha)$ is a solution of (1.14), then

$$
q(z ; \alpha)=\frac{4 \sigma^{\prime \prime}(z ; \alpha)+2 \alpha+1}{8 \sigma^{\prime}(z ; \alpha)}, \quad p(z ; \alpha)=-2 \sigma^{\prime}(z ; \alpha),
$$

with $^{\prime} \equiv \mathrm{d} / \mathrm{d} z$, are solutions of $(1.2)$ and $(1.21)$, respectively [84, 115, 116, 117].

In this paper some open problems associated with the Painlevé equations are discussed. Specifically the following open problems are discussed.

1. Develop algorithmic procedures for the Painlevé equivalence problem: given an equation with the Painlevé property, how do we know if the equation can be solved in terms of a Painlevé equation (or a Painlevé $\sigma$-equation)?

2. Develop software for numerically studying the Painlevé equations which utilizes the fact that they are integrable equations solvable using isomonodromy methods.

3. Develop a notation for the Painlevé transcendents which takes into account the wide variety of solutions the Painlevé equations have.

4. Provide a complete classification and unified structure of the special properties which the Painlevé equations possess - the presently known results are rather fragmentary and non-systematic.

\section{Painlevé equivalence problem}

For a linear ordinary differential equation, if it can be solved in terms of known functions then the equation is regarded as being is solved. Symbolic software such as MAPLE can easily find the solutions of the linear ordinary differential equations, as illustrated in the following example.

Example 2.1. Consider the linear equations

$$
\frac{\mathrm{d}^{2} v}{\mathrm{~d} z^{2}}+z^{2} v=0, \quad \frac{\mathrm{d}^{2} w}{\mathrm{~d} z^{2}}+\mathrm{e}^{2 z} w=0,
$$

which respectively have the solutions

$$
v(z)=\sqrt{z}\left\{C_{1} J_{1 / 4}\left(\frac{1}{2} z^{2}\right)+C_{2} J_{-1 / 4}\left(\frac{1}{2} z^{2}\right)\right\}, \quad w(z)=C_{1} J_{0}\left(\mathrm{e}^{z}\right)+C_{2} Y_{0}\left(\mathrm{e}^{z}\right),
$$

with $C_{1}$ and $C_{2}$ arbitrary constants, $J_{\nu}(\zeta)$ and $Y_{\nu}(\zeta)$ Bessel functions.

It is a general property of linear ordinary differential equations that all singularities of their solutions are fixed. For example, solutions of the second-order equation

$$
\frac{\mathrm{d}^{2} w}{\mathrm{~d} z^{2}}+p(z) \frac{\mathrm{d} w}{\mathrm{~d} z}+q(z) w=0
$$

can only have singularities where the coefficients do, namely at the singularities of $p(z)$ and $q(z)$. 
Definition 2.2. A fixed singular point of a solution of an ordinary differential equation is a singular point whose location does not vary with the particular solution chosen but depends only on the equation.

However it is not as simple for nonlinear ordinary differential equations which are quite different since, in general, their solutions can have both movable and fixed singularities.

Definition 2.3. A movable singular point of a solution of an ordinary differential equation is one whose location depends on the constant(s) of integration.

Currently there is no symbolic software available even to identify a nonlinear ordinary differential equation let alone find a solution, except for a few very simple examples. It is quite straightforward to determine whether a given (nonlinear) ordinary differential equation has the Painlevé property, e.g., using the Painlevé test [2, 3]; see also [1, 51, 98, 99].

Painlevé, Gambier et al. classified all ordinary differential equations of the form (1.7) with the Painlevé property, up to a Möbius transformation (1.8). Consequently, a given equation of the form (1.7) with the Painlevé property which is not in the list of fifty equations given by Ince [81, Chapter 14], how does one determine the Möbius transformation? If the equation is autonomous, or has a symmetry, then it has a first integral and one should be able to solve it in terms of elliptic equations, linear equations or by quadratures. If the equation is non-autonomous and does not possess a symmetry then it is likely to be solvable in terms of a Painlevé transcendent. The question is then to which one of the Painlevé equations (1.1)-(1.6) is the equation solvable in terms of?

We note that the solutions of some of the equations in the list given by Ince [81, Chapter 14] are solved in terms of Painlevé transcendents. For example, equation XX in the list, namely

$$
\frac{\mathrm{d}^{2} u}{\mathrm{~d} z^{2}}=\frac{1}{2 u}\left(\frac{\mathrm{d} u}{\mathrm{~d} z}\right)^{2}+4 u^{2}+z u,
$$

is solvable in terms of $\mathrm{P}_{\mathrm{II}}$ since letting $u(z)=\sqrt{w(z)}$ yields (1.2) with $\alpha=0$.

Example 2.4. Consider the equation

$$
\frac{\mathrm{d}^{2} w}{\mathrm{~d} z^{2}}=\frac{1}{w}\left(\frac{\mathrm{d} w}{\mathrm{~d} z}\right)^{2}-\frac{1}{z} \frac{\mathrm{d} w}{\mathrm{~d} z}+w^{3}-1 .
$$

This equation can be shown to possess the Painlevé property, but is not in the list of fifty equations given in [81, Chapter 14]. Equation (2.1) arises from the symmetry reduction

$$
u(x, t)=\ln w(z), \quad z=2 \sqrt{x t},
$$

of the Tzitzéica equation $[135,136,137]$

$$
u_{x t}=\mathrm{e}^{2 u}-\mathrm{e}^{-u}
$$

see also $[64,103,104,146]$. Making the transformation

$$
w(z)=x^{1 / 3} y(x), \quad z=\frac{3}{2} x^{2 / 3},
$$

in equation (2.1) yields

$$
\frac{\mathrm{d}^{2} y}{\mathrm{~d} x^{2}}=\frac{1}{y}\left(\frac{\mathrm{d} y}{\mathrm{~d} x}\right)^{2}-\frac{1}{x} \frac{\mathrm{d} y}{\mathrm{~d} x}+y^{3}-\frac{1}{x}
$$


which is the special case of $\mathrm{P}_{\text {III }}^{(7)}(1.11)$ with $\alpha=0$. The transformation (2.2) is suggested by the asymptotic expansions of (2.1) and (2.3)

$$
\begin{array}{llrl}
w(z) & \sim 1+\lambda z^{-1 / 2} \exp (-\sqrt{3} z), & & \text { as } \quad z \rightarrow \infty, \\
y(x) & \sim x^{-1 / 3}+\kappa x^{-2 / 3} \exp \left(-\frac{3}{2} \sqrt{3} x^{2 / 3}\right), & \text { as } \quad x \rightarrow \infty,
\end{array}
$$

with $\lambda$ and $\kappa$ constants. Consequently one can derive the isomonodromy problem for equation (2.1) from that of equation (2.3).

Example 2.5. Consider the complex sine-Gordon equation

$$
\nabla^{2} \psi+\frac{(\nabla \psi)^{2} \bar{\psi}}{1-|\psi|^{2}}+\psi\left(1-|\psi|^{2}\right)=0
$$

where $\nabla \psi=\left(\psi_{x}, \psi_{y}\right)$, which is also known as the Pohlmeyer-Lund-Regge model [101, 102, 125]. This has a separable solution in polar coordinates given by $\psi(r, \theta)=\varphi_{n}(r) \mathrm{e}^{\mathrm{i} n \theta}$, where $\varphi_{n}(r)$ satisfies the second-order equation

$$
\frac{\mathrm{d}^{2} \varphi_{n}}{\mathrm{~d} r^{2}}+\frac{1}{r} \frac{\mathrm{d} \varphi_{n}}{\mathrm{~d} r}+\frac{\varphi_{n}}{1-\varphi_{n}^{2}}\left\{\left(\frac{\mathrm{d} \varphi_{n}}{\mathrm{~d} r}\right)^{2}-\frac{n^{2}}{r^{2}}\right\}+\varphi_{n}\left(1-\varphi_{n}^{2}\right)=0,
$$

which also arises in extended quantum systems [37, 38, 39], in relativity [74] and reflection coefficients for orthogonal polynomials on the unit circle [140, equation (3.13)]. Equation (2.5) can be shown to possess the Painleve property, though is not in the list of 50 equations given in [81, Chapter 14]. Equation (2.5) can be transformed into $\mathrm{P}_{\mathrm{V}}$ (1.5) in two different ways:

(i) the transformation

$$
\varphi_{n}(r)=\frac{1+u(z)}{1-u(z)}, \quad \text { with } \quad r=\frac{1}{2} z
$$

yields

$$
\frac{\mathrm{d}^{2} u}{\mathrm{~d} z^{2}}=\left(\frac{1}{2 u}+\frac{1}{u-1}\right)\left(\frac{\mathrm{d} u}{\mathrm{~d} z}\right)^{2}-\frac{1}{z} \frac{\mathrm{d} u}{\mathrm{~d} z}+\frac{n^{2}(u-1)^{2}\left(u^{2}-1\right)}{8 z^{2} u}-\frac{u(u+1)}{2(u-1)},
$$

which is $\mathrm{P}_{\mathrm{V}}(1.5)$ with $\alpha=\frac{1}{8} n^{2}, \beta=-\frac{1}{8} n^{2}, \gamma=0$ and $\delta=-\frac{1}{2}$;

(ii) the transformation

$$
\varphi_{n}(r)=\sqrt{\frac{v(z)}{v(z)-1}}, \quad \text { with } \quad r=\sqrt{z}
$$

yields

$$
\frac{\mathrm{d}^{2} v}{\mathrm{~d} z^{2}}=\left(\frac{1}{2 v}+\frac{1}{v-1}\right)\left(\frac{\mathrm{d} v}{\mathrm{~d} z}\right)^{2}-\frac{1}{z} \frac{\mathrm{d} v}{\mathrm{~d} z}+\frac{n^{2} v(v-1)^{2}}{2 z^{2}}-\frac{v}{2 z},
$$

which is $\mathrm{P}_{\mathrm{V}}$ (1.5) with $\alpha=\frac{1}{2} n^{2}, \beta=0, \gamma=-\frac{1}{2}$ and $\delta=0$, i.e., deg- $\mathrm{P}_{\mathrm{V}}$ (1.12).

It is known that deg- $\mathrm{P}_{\mathrm{V}}$ (1.12) is equivalent to $\mathrm{P}_{\mathrm{III}}$ (1.9), cf. [76, Section 34]. Using this it can be shown that if $w(z)$ satisfies

$$
\frac{\mathrm{d}^{2} w}{\mathrm{~d} z^{2}}=\frac{1}{w}\left(\frac{\mathrm{d} w}{\mathrm{~d} z}\right)^{2}-\frac{1}{z} \frac{\mathrm{d} w}{\mathrm{~d} z}-\frac{2 n w^{2}}{z}+\frac{2 n+2}{z}+\gamma w^{3}+\frac{\delta}{w},
$$


which is $\mathrm{P}_{\mathrm{III}}(1.9)$ with $\alpha=-2 n$ and $\beta=2 n+2$, then

$$
\varphi_{n}(r)=\frac{\sqrt{-z w^{\prime}(z)+z w^{2}(z)+(2 n+1) w(z)+z}}{\sqrt{2 z} w(z)}, \quad \text { with } \quad r=z
$$

satisfies (2.5). Consequently solutions of equation (2.5) can be expressed in terms of solutions of both $\mathrm{P}_{\mathrm{III}}$ (1.3) and $\mathrm{P}_{\mathrm{V}}(1.5)$.

The function $\varphi_{n}(r)$ also satisfies the differential-difference equations

$$
\begin{aligned}
& \frac{\mathrm{d} \varphi_{n}}{\mathrm{~d} r}+\frac{n}{r} \varphi_{n}-\left(1-\varphi_{n}^{2}\right) \varphi_{n-1}=0, \\
& \frac{\mathrm{d} \varphi_{n-1}}{\mathrm{~d} r}-\frac{n-1}{r} \varphi_{n-1}+\left(1-\varphi_{n-1}^{2}\right) \varphi_{n}=0 .
\end{aligned}
$$

Solving (2.6a) for $\varphi_{n-1}(r)$ and substituting in (2.6b) yields equation (2.5), whilst eliminating the derivatives in (2.6), after letting $n \rightarrow n+1$ in (2.6b), yields the difference equation

$$
\varphi_{n+1}+\varphi_{n-1}=\frac{2 n}{r} \frac{\varphi_{n}}{1-\varphi_{n}^{2}},
$$

which is known as the discrete Painlevé II equation [108, 124, 140]. If $n=1$ then equations (2.6) have the solution

$$
\varphi_{0}(r)=1, \quad \varphi_{1}(r)=\frac{C_{1} I_{1}(r)-C_{2} K_{1}(r)}{C_{1} I_{0}(r)+C_{2} K_{0}(r)},
$$

where $I_{0}(r), K_{0}(r), I_{1}(r)$ and $K_{1}(r)$ are the imaginary Bessel functions and $C_{1}$ and $C_{2}$ are arbitrary constants. Then one can use (2.7) to determine $\varphi_{n}(r)$, for $n=2,3, \ldots$ Using this Barashenkov and Pelinovsky [20] derive explicit multi-vortex solutions for the complex sineGordon equation (2.4).

The relationship between solutions of (2.5) and those of $\mathrm{P}_{\mathrm{III}}$ (1.9), is illustrated in the following theorem.

Theorem 2.6. If $\varphi_{n}(r)$ satisfies (2.5) then $w_{n}(r)=\varphi_{n+1}(r) / \varphi_{n}(r)$ satisfies

$$
\frac{\mathrm{d}^{2} w_{n}}{\mathrm{~d} r^{2}}=\frac{1}{w_{n}}\left(\frac{\mathrm{d} w_{n}}{\mathrm{~d} r}\right)^{2}-\frac{1}{r} \frac{\mathrm{d} w_{n}}{\mathrm{~d} r}-\frac{2 n}{r} w_{n}^{2}+\frac{2 n+2}{r}+w_{n}^{3}-\frac{1}{w_{n}},
$$

which is $P_{\mathrm{III}}(1.3)$ with parameters $\alpha=-2 n$ and $\beta=2 n+2$.

Proof. See Hisakado [79] and Tracy and Widom [132].

Example 2.7. In their study of third-order ordinary differential equations, Muğan and Jrad [107] show that the equations

$$
\begin{aligned}
& y^{2} \frac{\mathrm{d}^{3} y}{\mathrm{~d} x^{3}}=4 y \frac{\mathrm{d} y}{\mathrm{~d} x} \frac{\mathrm{d}^{2} y}{\mathrm{~d} x^{2}}-3\left(\frac{\mathrm{d} y}{\mathrm{~d} x}\right)^{3}+y^{4} \frac{\mathrm{d} y}{\mathrm{~d} x}+4 \kappa \mu x\left(y \frac{\mathrm{d} y}{\mathrm{~d} x}-\kappa y^{3}\right)-4 \kappa \mu y^{2}+3 \mu \frac{\mathrm{d} y}{\mathrm{~d} x}, \\
& y \frac{\mathrm{d}^{3} y}{\mathrm{~d} x^{3}}=2 \frac{\mathrm{d} y}{\mathrm{~d} x} \frac{\mathrm{d}^{2} y}{\mathrm{~d} x^{2}}-2 y^{2} \frac{\mathrm{d}^{2} y}{\mathrm{~d} x^{2}}+y^{3} \frac{\mathrm{d} y}{\mathrm{~d} x}+y^{5}+\kappa\left(2 \frac{\mathrm{d} y}{\mathrm{~d} x}+x y^{3}+y^{2}\right), \\
& y \frac{\mathrm{d}^{3} y}{\mathrm{~d} x^{3}}=\frac{\mathrm{d} y}{\mathrm{~d} x} \frac{\mathrm{d}^{2} y}{\mathrm{~d} x^{2}}-2 y^{3}+\kappa y^{2}-\frac{\kappa^{2}}{12}\left(x \frac{\mathrm{d} y}{\mathrm{~d} x}-y\right),
\end{aligned}
$$

where $\kappa$ and $\mu$ are non-zero constants, have the Painlevé property. In [107] see equation (2.67) with $k_{1}=3 \mu, k_{2}=0$, without loss of generality, and $k_{3}=4 \kappa \mu$; equation (2.106) with $k_{2}=\kappa$ 
and $k_{3}=0$, without loss of generality; and equation (4.14) with $k_{1}=\kappa$ and $k_{2}=0$, without loss of generality ${ }^{1}$. Levi, Sekera and Winternitz [100] show that (2.8), (2.10) and (2.10) have no symmetries and state that these equations are "candidates for new Painlevé transcendents"; see equations (3.3), (3.4) and (3.4) in [100]. However, we show below, equation (2.8) can be solved in terms of $\mathrm{P}_{\mathrm{IV}}$ (1.4) and equation (2.9) and (2.10) in terms of $\mathrm{P}_{34}$ (1.21).

Letting $y=\frac{1}{u} \frac{\mathrm{d} u}{\mathrm{~d} x}$ in equation (2.8) gives the tri-linear equation

$$
\begin{gathered}
\left(\frac{\mathrm{d} u}{\mathrm{~d} x}\right)^{2} \frac{\mathrm{d}^{4} u}{\mathrm{~d} x^{4}}-4 \frac{\mathrm{d} u}{\mathrm{~d} x} \frac{\mathrm{d}^{2} u}{\mathrm{~d} x^{2}} \frac{\mathrm{d}^{3} u}{\mathrm{~d} x^{3}}+3\left(\frac{\mathrm{d}^{2} u}{\mathrm{~d} x^{2}}\right)^{3}+\left(4 \kappa \mu x u \frac{\mathrm{d} u}{\mathrm{~d} x}+3 \mu u^{2}\right) \frac{\mathrm{d}^{2} u}{\mathrm{~d} x^{2}} \\
+4 \kappa \mu(\kappa+1) x\left(\frac{\mathrm{d} u}{\mathrm{~d} x}\right)^{3}-\mu(4 \kappa+3) u\left(\frac{\mathrm{d} u}{\mathrm{~d} x}\right)^{2}=0,
\end{gathered}
$$

which has the first integral, the bi-linear equation

$$
\left(\frac{\mathrm{d} u}{\mathrm{~d} x}\right) \frac{\mathrm{d}^{3} u}{\mathrm{~d} x^{3}}=\frac{3}{2}\left(\frac{\mathrm{d}^{2} u}{\mathrm{~d} x^{2}}\right)^{2}-\left(2 \kappa^{2} \mu x^{2}+K\right)\left(\frac{\mathrm{d} u}{\mathrm{~d} x}\right)^{2}-4 \kappa \mu x u \frac{\mathrm{d} u}{\mathrm{~d} x}-\frac{3}{2} \mu u^{2},
$$

with $K$ a constant of integration. Since $\frac{\mathrm{d} u}{\mathrm{~d} x}=u y$, then we obtain the second-order ordinary differential equation

$$
\frac{\mathrm{d}^{2} y}{\mathrm{~d} x^{2}}=\frac{3}{2 y}\left(\frac{\mathrm{d} y}{\mathrm{~d} x}\right)^{2}+\frac{1}{2} y^{3}-\left(2 \kappa^{2} \mu x^{2}-K\right) y-4 \kappa \mu x-\frac{3 \mu}{2 y},
$$

which is the first integral of (2.8) and is not one of the 50 equations in Ince's list. However, making the transformation

$$
y(x)=\frac{\mu^{1 / 4}}{\kappa^{1 / 2} w(z)}, \quad z=\kappa^{1 / 2} \mu^{1 / 4} x
$$

yields $\mathrm{P}_{\mathrm{IV}}$ (1.4) with parameters

$$
\alpha=\frac{K}{\kappa \mu^{1 / 2}}, \quad \beta=-\frac{1}{2 \kappa^{2}} .
$$

Letting $y=\frac{1}{u} \frac{\mathrm{d} u}{\mathrm{~d} x}$ in equation (2.9) gives the tri-linear equation

$$
u \frac{\mathrm{d} u}{\mathrm{~d} x} \frac{\mathrm{d}^{4} u}{\mathrm{~d} x^{4}}=\left[u \frac{\mathrm{d}^{2} u}{\mathrm{~d} x^{2}}+\left(\frac{\mathrm{d} u}{\mathrm{~d} x}\right)^{2}\right] \frac{\mathrm{d}^{3} u}{\mathrm{~d} x^{3}}+2 \kappa u \frac{\mathrm{d}^{2} u}{\mathrm{~d} x^{2}}+\kappa x\left(\frac{\mathrm{d} u}{\mathrm{~d} x}\right)^{3}-\kappa u\left(\frac{\mathrm{d} u}{\mathrm{~d} x}\right)^{2},
$$

which has the first integral

$$
\frac{\mathrm{d}^{3} u}{\mathrm{~d} x^{3}}+\left(\kappa x-3 C_{1} u\right) \frac{\mathrm{d} u}{\mathrm{~d} x}+2 \kappa u=0,
$$

with $C_{1}$ a constant of integration. Letting $u=v+\kappa x / C_{1}$ gives

$$
\frac{\mathrm{d}^{3} v}{\mathrm{~d} x^{3}}-3 C_{1} v \frac{\mathrm{d} v}{\mathrm{~d} x}=2 \kappa x \frac{\mathrm{d} v}{\mathrm{~d} x}+\kappa v .
$$

\footnotetext{
${ }^{1}$ The sign of the last term in (2.10) has been changed as there is a sign error in [107, equation (4.14)].
} 
Multiplying this by $v$ and integrating gives

$$
v \frac{\mathrm{d}^{2} v}{\mathrm{~d} x^{2}}=\frac{1}{2}\left(\frac{\mathrm{d} v}{\mathrm{~d} x}\right)^{2}+C_{1} v^{3}+\kappa x v^{2}+C_{2},
$$

with $C_{2}$ a constant of integration, which is equivalent to $\mathrm{P}_{34}(1.21)$, by rescaling the variables if necessary. Due to the relationship between $\mathrm{P}_{34}$ (1.21) and $\mathrm{P}_{\mathrm{II}}$ (1.2) [67], solutions of equation (2.9) can be expressed in terms of solutions of $\mathrm{P}_{\mathrm{II}}(1.2)$. Specifically if $w(z)$ is a solution of $\mathrm{P}_{\mathrm{II}}(1.2)$, then

$$
y(x)=-\kappa^{1 / 3}\left\{2 w(z)+\frac{z w(z)+\alpha}{w^{\prime}(z)+w^{2}(z)}\right\}, \quad x=-z / \kappa^{1 / 3},
$$

where $^{\prime} \equiv \mathrm{d} / \mathrm{d} z$ satisfies (2.9).

Letting $y=\frac{\mathrm{d} u}{\mathrm{~d} x}$ in equation (2.10) and integrating gives the third-order equation

$$
\frac{\mathrm{d}^{3} u}{\mathrm{~d} x^{3}}+(24 u-\kappa x) \frac{\mathrm{d} u}{\mathrm{~d} x}-\frac{\kappa^{2} x}{12}=0,
$$

where the constant of integration has been set to zero, without loss of generality. Then making the transformation

$$
u=-\frac{1}{4} v-\frac{1}{24} \kappa x,
$$

yields

$$
\frac{\mathrm{d}^{3} v}{\mathrm{~d} x^{3}}=(6 v+2 \kappa x) \frac{\mathrm{d} v}{\mathrm{~d} x}+\kappa v .
$$

Multiplying this by $v$ and integrating gives

$$
v \frac{\mathrm{d}^{2} v}{\mathrm{~d} x^{2}}=\frac{1}{2}\left(\frac{\mathrm{d} v}{\mathrm{~d} x}\right)^{2}+2 v^{3}+\kappa x v^{2}+C
$$

with $C$ a constant of integration, which is equivalent to $\mathrm{P}_{34}(1.21)$, by rescaling the variables if necessary.

Remark 2.8. We remark that equations (2.11) and (2.12), after rescaling the variables, arise as a scaling reduction of the Korteweg-de Vries equation [67] and as a nonclassical reduction of the Boussinesq equation [46].

Bureau [35] (see also [36, 40]) has also studied the classification of second order, second degree equations

$$
\left(\frac{\mathrm{d}^{2} w}{\mathrm{~d} z^{2}}\right)^{2}=F\left(w, \frac{\mathrm{d} w}{\mathrm{~d} z}, z\right)+G\left(w, \frac{\mathrm{d} w}{\mathrm{~d} z}, z\right) \frac{\mathrm{d}^{2} w}{\mathrm{~d} z^{2}}
$$

where $F$ and $G$ are rational in $w$ and $\mathrm{d} w / \mathrm{d} z$ and locally analytic in $z$. Cosgrove and Scoufis [58] have classified all equations with the Painlevé property for the special case of $(2.13)$ when $G \equiv 0$, i.e.,

$$
\left(\frac{\mathrm{d}^{2} w}{\mathrm{~d} z^{2}}\right)^{2}=F\left(w, \frac{\mathrm{d} w}{\mathrm{~d} z}, z\right)
$$


where $F$ is rational in $w$ and $\mathrm{d} w / \mathrm{d} z$, locally analytic in $z$ and not a perfect square. Cosgrove and Scoufis [58] solved the equations with the Painlevé property in terms of the Painlevé transcendents, elliptic functions, and solutions of linear equations, see also [53, 57, 129, 130]. Cosgrove [52] classified all equations that are of Painlevé type of the form

$$
\left(\frac{\mathrm{d}^{2} w}{\mathrm{~d} z^{2}}\right)^{m}=F\left(w, \frac{\mathrm{d} w}{\mathrm{~d} z}, z\right), \quad m \geq 3,
$$

where $F$ is rational in $w$ and $\mathrm{d} w / \mathrm{d} z$ and locally analytic in $z$ and solved the equations in terms of the first, second and fourth Painlevé transcendents, elliptic functions, or quadratures.

For various results on classifying classes of second-order ordinary differential equations, including Painlevé equations, see Babich and Bordag [12], Bagderina [13, 15, 16, 17, 18], Bagderina and Tarkhanov [19], Berth and Czichowski [22], Hietarinta and Dryuma [78], Kamran, Lamb and Shadwick [88], Kartak [90, 91, 92, 93], Kossovskiy and Zaitsev [97], Milson and Valiquette [106], Valiquette [139] and Yumaguzhin [145]. Most of these studies are concerned with the invariance of second-order ordinary differential equations of the form

$$
\frac{\mathrm{d}^{2} w}{\mathrm{~d} z^{2}}=F_{3}(w, z)\left(\frac{\mathrm{d} w}{\mathrm{~d} z}\right)^{3}+F_{2}(w, z)\left(\frac{\mathrm{d} w}{\mathrm{~d} z}\right)^{2}+F_{1}(w, z) \frac{\mathrm{d} w}{\mathrm{~d} z}+F_{0}(w, z),
$$

under the point transformations of the form

$$
w=\psi(y, x), \quad z=\phi(y, x), \quad \frac{\partial(\psi, \phi)}{\partial(y, x)} \equiv \frac{\partial \psi}{\partial y} \frac{\partial \phi}{\partial x}-\frac{\partial \psi}{\partial x} \frac{\partial \phi}{\partial y} \neq 0 .
$$

Chazy [41, 42], Garnier [75] and Bureau [34] have obtained partial results on the classification of ordinary differential equations with the Painlevé property for third-order equations of the form

$$
\frac{\mathrm{d}^{3} w}{\mathrm{~d} z^{3}}=F\left(w, \frac{\mathrm{d} w}{\mathrm{~d} z}, \frac{\mathrm{d}^{2} w}{\mathrm{~d} z^{2}}, z\right)
$$

where $F$ is rational in $w$ and its derivatives, and locally analytic in $z$. Despite the considerable length of these papers, only a very small proportion of the possible equations with the Painlevé property in each class were discovered. Further no new transcendents were discovered, i.e., every equation with the Painlevé property was shown to be solvable in terms of previously known equations, either Painlevé transcendents, elliptic functions or quadratures.

Most of the recent studies of Painlevé classification for third-order equations have concentrated on equations in the Bureau polynomial class where the function $F$ in (2.14) is polynomial in $w$ and its derivatives, rather than rational. Cosgrove $[55,56]$ classified third-order equations of this specific form with the Painlevé property and solved the equations in terms of the Painlevé transcendents, elliptic functions, solutions of linear equations or quadratures; see also [14, 54].

Open Problem 2.9. Given an ordinary differential equation with the Painlevé property, how do we know whether it can be solved in terms of a Painlevé transcendent?

\section{Notation for Painlevé transcendents}

Uniquely amongst the functions discussed in the DLMF [118], there is no special notation for the Painlevé transcendents, i.e., the solutions of the Painlevé equations. There are several functions in the DLMF whose notation involves $P$, or a variant, e.g., $P_{n}^{(\alpha, \beta)}(z)$ (Jacobi polynomials), $P_{n}(z)$ (Legendre polynomials), and $\wp(z)$ (Weierstrass elliptic functions). For linear equations, there are a finite number of linearly independent solutions, e.g., $\operatorname{Ai}(z)$ and $\operatorname{Bi}(z)$ for the Airy equation

$$
\frac{\mathrm{d}^{2} w}{\mathrm{~d} z^{2}}-z w=0 .
$$


However, for nonlinear equations such as the Painlevé equations, the issue of notation is not as simple as there are numerous completely different solutions. Although second-order equations, there don't exist two "representative solutions". What is needed is some agreed notation for the Painlevé transcendents. In fact, unlike the linear case when the set of all solutions is a finite dimensional vector space, the set of all solutions of a Painlevé equation form a transcendental structure (a foliation travelling through a fibre bundle, each fibre of which is described by an affine Dynkin diagram) without any global coordinates which could be used as natural universal markers of the solutions. Such a notation would assist in the classification of properties of Painlevé equations.

For example, there are several different types of solutions of $\mathrm{P}_{\mathrm{II}}(1.2)$.

(i) The general solution of $\mathrm{P}_{\mathrm{II}}$ is a transcendental function for all values of $\alpha$ and involves two arbitrary constants.

(ii) Suppose that $w_{k}(z)$ is the solution of $\mathrm{P}_{\text {II }}$ with $\alpha=0$, i.e.,

$$
\frac{\mathrm{d}^{2} w_{k}}{\mathrm{~d} z^{2}}=2 w_{k}^{3}+z w_{k}
$$

with the asymptotic behaviour

$$
w_{k}(z) \sim k \operatorname{Ai}(z), \quad \text { as } \quad z \rightarrow \infty,
$$

where $k$ is a real parameter and $\operatorname{Ai}(z)$ is the Airy function, which uniquely determines the solution. This family of solutions has different analytical properties on the real axis and have different asymptotic behaviours as $z \rightarrow-\infty$, depending on the parameter $k$.

- If $|k|<1$, then $w_{k}(z)$ is the Ablowitz-Segur solution [4, 131], which is pole-free on the real axis and as $z \rightarrow-\infty$ has oscillatory behaviour with algebraic decay given by

$$
w_{k}(z)=d|z|^{-1 / 4} \sin \left(\frac{2}{3}|z|^{3 / 2}-\frac{3}{4} d^{2} \ln |z|-\theta_{0}\right)+o\left(|z|^{-1 / 4}\right),
$$

where the connection formulae $d^{2}(k)$ and $\theta_{0}(k)$, which relate the asymptotic behaviours (3.1) and (3.2) as $z \rightarrow \pm \infty$, are

$$
\begin{aligned}
& d^{2}(k)=-\pi^{-1} \ln \left(1-k^{2}\right), \\
& \theta_{0}(k)=\frac{3}{2} d^{2} \ln 2+\arg \left\{\Gamma\left(1-\frac{1}{2} \mathrm{i} d^{2}\right)\right\}+\frac{1}{4} \pi[1-2 \operatorname{sgn}(k)],
\end{aligned}
$$

see $[21,48,63]$.

- If $k= \pm 1$ then $w_{k}(z)$ is the Hastings-McLeod solution [77] which is monotonic, polefree on the real axis and has algebraic growth as $z \rightarrow-\infty$ given by

$$
w_{ \pm 1}(z)= \pm\left(\frac{1}{2}|z|\right)^{1 / 2}+o\left(|z|^{1 / 2}\right) .
$$

- If $|k|>1$ then $w_{k}(z)$ is a singular solution which has infinitely many poles on the negative real axis - see the numerical plot by Fornberg and Weideman [70, Fig. 12] and has singular oscillatory behaviour as $z \rightarrow-\infty$ given by

$$
w_{k}(z)=\frac{\sqrt{|z|}}{\sin \left\{\frac{2}{3}|z|^{3 / 2}+\beta \ln \left(8|z|^{3 / 2}\right)+\phi\right\}+\mathcal{O}\left(|z|^{-3 / 2}\right)}+\mathcal{O}\left(|z|^{-1}\right),
$$

where $z$ bounded away from the singularities appearing in the denominator and the connection formulae $\beta(k)$ and $\phi(k)$ are

$$
\beta(k)=\frac{1}{2} \pi^{-1} \ln \left(k^{2}-1\right), \quad \phi(k)=-\arg \left[\Gamma\left(\frac{1}{2} \mathrm{i} \beta\right)\right]+\frac{1}{2} \pi[\operatorname{sgn}(k)-1],
$$

see $[29,89]$. 
- Bothner [28] discusses the transition from the Ablowitz-Segur solution (3.2) and the singular solution (3.4) to the Hastings-McLeod solution (3.3) as $z \rightarrow-\infty$ and $|k| \rightarrow 1$. The transition asymptotics are expressed in terms of the Jacobi elliptic functions.

- The case when $k=\mathrm{i} \kappa$, with $\kappa \in \mathbb{R}$, in the boundary condition (3.1), known as the pure imaginary Ablowitz-Segur solution, is discussed by Its and Kapaev [82].

- Bogatskiy, Claeys and Its [25] extended these results to discuss complex AblowitzSegur solutions in the case when $k \in \mathbb{C}$.

(iii) For $\mathrm{P}_{\mathrm{II}}$ with $\alpha \neq 0$, there are analogs of the Ablowitz-Segur and Hastings-McLeod solutions, known as the quasi-Ablowitz-Segur solution and the quasi-Hastings-McLeod solution $[43,59,60]$; see also $[49,70,72,134]$. There is an extensive literature regarding the asymptotics for $\mathrm{P}_{\mathrm{II}}(1.2)$ when $\alpha=0$. There are significantly fewer asymptotic results in the case when $\alpha \neq 0$. Further, whilst the Ablowitz-Segur and Hastings-McLeod solutions have exponential decay as $z \rightarrow \infty$ given by (3.1), when $\alpha \neq 0$ then the solutions only have algebraic decay given by

$$
w(z ; \alpha) \sim-\alpha / z, \quad \text { as } \quad z \rightarrow \infty .
$$

For the quasi-Ablowitz-Segur solution when $\alpha \in\left(-\frac{1}{2}, \frac{1}{2}\right)$, there exists a one-parameter family of real solutions $w(z)$ for $k \in(-\cos (\pi \alpha), \cos (\pi \alpha))$ with the following properties:

$$
w(z)=B(z ; \alpha)+k \operatorname{Ai}(z)\left[1+\mathcal{O}\left(z^{-3 / 4}\right)\right], \quad \text { as } \quad z \rightarrow \infty,
$$

and

$$
w(z)=d|z|^{-1 / 4} \cos \left(\frac{2}{3}|z|^{3 / 2}-\frac{3}{4} d^{2} \ln |z|+\phi\right)+\mathcal{O}\left(|z|^{-1}\right), \quad \text { as } \quad z \rightarrow-\infty,
$$

where $\operatorname{Ai}(z)$ is the Airy function and $B(z ; \alpha)$ is given by

$$
B(z ; \alpha) \sim \frac{\alpha}{z} \sum_{n=0}^{\infty} \frac{a_{n}}{z^{3 n}}
$$

with coefficients $a_{n}$ which are uniquely determined by the recurrence relation

$$
a_{n+1}=(3 n+1)(3 n+2) a_{n}-2 \alpha^{2} \sum_{j, k, \ell=0}^{n} a_{j} a_{k} a_{\ell}, \quad a_{0}=1 .
$$

The connection formulas are given by

$$
\begin{aligned}
& d(k)=\pi^{-1 / 2} \sqrt{-\ln \left(\cos ^{2}(\pi \alpha)-k^{2}\right),} \\
& \phi(k)=-\frac{3}{2} d^{2} \ln 2+\arg \Gamma\left(\frac{1}{2} \mathrm{i} d^{2}\right)-\frac{1}{4} \pi-\arg (-\sin (\pi \alpha)-k \mathrm{i}),
\end{aligned}
$$

see Dai and $\mathrm{Hu}[59,60]$. For the quasi-Hastings-McLeod solution, Claeys, Kuijlaars and Vanlessen [43] show that there exists a unique solution which is pole-free on the real axis with the asymptotic behaviours

$$
\begin{array}{ll}
w(z) \sim-\alpha / z, & z \rightarrow+\infty, \\
w(z) \sim \sqrt{\frac{1}{2}|z|,} & z \rightarrow-\infty ;
\end{array}
$$

see also $[59,60]$. 
(iv) Special function solutions of $\mathrm{P}_{\mathrm{II}}$ arise if and only if $\alpha=n+\frac{1}{2}$, with $n \in \mathbb{Z}$, which involve one arbitrary constant [73]. These are expressed in terms of the $n \times n$ Wronskian determinant

$$
\tau_{n}(z ; \vartheta)=\operatorname{det}\left[\frac{\mathrm{d}^{j+k}}{\mathrm{~d} z^{j+k}} \varphi(z ; \vartheta)\right]_{j, k=0}^{n-1}, \quad n \geq 1,
$$

where

$$
\varphi(z ; \vartheta)=\cos (\vartheta) \operatorname{Ai}(\zeta)+\sin (\vartheta) \operatorname{Bi}(\zeta), \quad \zeta=-2^{-1 / 3} z,
$$

with $\operatorname{Ai}(\zeta)$ and $\operatorname{Bi}(\zeta)$ the Airy functions and $\vartheta$ an arbitrary constant; see also the recent studies [45, 61].

(v) Rational solutions of $\mathrm{P}_{\mathrm{II}}$ exist if and only if $\alpha=n$, with $n \in \mathbb{Z}$, which involve no arbitrary constants $[141,143]$. These solutions are expressed in terms of polynomials $Q_{n}(z)$ of degree $\frac{1}{2} n(n+1)$, now known as the Yablonskii-Vorob'ev polynomials, which are defined through the recurrence relation (a second-order, bilinear differential-difference equation)

$$
Q_{n+1} Q_{n-1}=z Q_{n}^{2}-4\left[Q_{n} \frac{\mathrm{d}^{2} Q_{n}}{\mathrm{~d} z^{2}}-\left(\frac{\mathrm{d} Q_{n}}{\mathrm{~d} z}\right)^{2}\right],
$$

with $Q_{0}(z)=1$ and $Q_{1}(z)=z$. Clarkson and Mansfield [47] investigated the locations of the roots of the Yablonskii-Vorob'ev polynomials in the complex plane and showed that these roots have a very regular, approximately triangular structure; the term "approximate" is used since the patterns are not exact triangles as the roots lie on arcs rather than straight lines. Bertola and Bothner [24] and Buckingham and Miller [32, 33] have studied the Yablonskii-Vorob'ev polynomials $Q_{n}(z)$ in the limit as $n \rightarrow \infty$ and shown that the roots lie in a "triangular region" with elliptic sides which meet with interior angle $\frac{2}{5} \pi$.

(vi) There exist tronquée and tri-tronquée solutions of $\mathrm{P}_{\mathrm{II}}$, which are pole-free in sectors of the complex plane [30, 31]; see also [23, 80, 85, 86, 105, 111].

Open Problem 3.1. Develop a notation for the Painlevé transcendents which takes into account the wide variety of solutions the Painlevé equations have.

\section{Numerical solution of Painlevé equations}

Numerical analysis of the Painlevé equations presents novel challenges: in particular, in contrast to the classical special functions, where the linearity of the equations greatly simplifies the situation, each problem for the nonlinear Painlevé equations arises essentially anew. Ideally what is needed is reliable, easy to use software to compute numerically the solutions of the Painlevé equations. On the other hand, Painlevé transcendents, being solutions of integrable nonlinear equations, have much global information available about them. The software should be in the form of a living document where new numerical problems can be addressed by a pool of experts as they arise, as well as providing access to existing software. At the technical level, how does one combine asymptotic information about the solutions obtained from the RiemannHilbert problem, together with efficient numerical codes in order to compute the solution $w(z)$ at finite values of $z$ ? A comprehensive analysis presents many challenges, conceptual, philosophical and technical.

Deift [62] wrote:

Writing useful numerical software for such nonlinear equations [i.e., the Painlevé equations] presents many challenges, conceptual, philosophical and technical. Without the help of linearity, it is not at all clear how to select a broad enough class of "representative problems". 
Numerical simulations of the Painlevé equations given in [44, 45] were obtained using MAPLE using the DEplot command with option method=dverk78, which finds a numerical solution using a seventh-eighth order continuous Runge-Kutta method. This is relatively simple to use, gives plots of solutions quickly with accuracy better than the human eye can detect, and generally works fine for initial value problems.

Some recent numerical computations of Painlevé equations include: a pole field solver using Padé approximations [65, 66, 69, 70, 71, 126, 127]; numerical Riemann-Hilbert problems [119, 120, 122, 121, 133, 142]; Fredholm determinants [26, 27]; Padé approximations [110, 112, 144]; pole elimination $[5,6,7,8,9,10,11]$; a multidomain spectral method [96].

\section{Open Problem 4.1.}

- The Runge-Kutta method, and variants, are standard ODE solvers. Can we do better for integrable equations such as the Painlevé equations?

- Painlevé equations are "integrable" and solvable by the isomonodromy method through an associated Riemann-Hilbert problem. How can we use this in the development of software for studying the Painlevé equations numerically?

- It is well known that there are discrete Painlevé equations, which are integrable discrete equations that tend to the associated Painlevé equations in an appropriate continuum limit. Should we use a "integrable discretization" of the Painlevé equations?

\section{Classification of properties of Painlevé equations}

The Painlevé equations are known to have a cornucopia of properties such as: a Hamiltonian representation; exact solutions (rational solutions, algebraic solutions, solutions in terms of classical special solutions); Bäcklund transformations (which relate two solutions of a Painlevé equation); associated isomonodromy problems (which are Lax pairs that express the Painlevé equation as the compatibility of two linear systems); and asymptotic approximations in the complex plane, with associated connection formulae relating the asymptotics. For details see $[44,50,68,76,83,87,109]$ and the references therein.

Open Problem 5.1. A complete classification and a unifying structure for these properties is needed as the presently known results are rather fragmentary and non-systematic.

\section{Acknowledgments}

I would like to thank Mark Ablowitz, Andrew Bassom, Chris Cosgrove, Alfredo Deaño, Percy Deift, Marco Fasondini, Bengt Fornberg, David Gómez-Ullate, Rod Halburd, Andrew Hone, Alexander Its, Kerstin Jordaan, Nalini Joshi, Erik Koelink, Martin Kruskal, Ana Loureiro, Elizabeth Mansfield, Marta Mazzocco, Bryce McLeod, Peter Miller, Walter Van Assche, and André Weideman for their helpful comments and illuminating discussions.

\section{References}

[1] Ablowitz M.J., Clarkson P.A., Solitons, nonlinear evolution equations and inverse scattering, London Mathematical Society Lecture Note Series, Vol. 149, Cambridge University Press, Cambridge, 1991.

[2] Ablowitz M.J., Ramani A., Segur H., Nonlinear evolution equations and ordinary differential equations of Painlevé type, Lett. Nuovo Cimento 23 (1978), 333-338.

[3] Ablowitz M.J., Ramani A., Segur H., A connection between nonlinear evolution equations and ordinary differential equations of P-type. I, J. Math. Phys. 21 (1980), 715-721. 
[4] Ablowitz M.J., Segur H., Exact linearization of a Painlevé transcendent, Phys. Rev. Lett. 38 (1977), 11031106.

[5] Abramov A.A., Yukhno L.F., Numerical solution of the Cauchy problem for Painlevé III, Differ. Equ. 48 (2012), 909-918.

[6] Abramov A.A., Yukhno L.F., Numerical solution of the Cauchy problem for the Painlevé I and II equations, Comput. Math. Math. Phys. 52 (2012), 321-329.

[7] Abramov A.A., Yukhno L.F., Numerical solution of the Painlevé IV equation, Comput. Math. Math. Phys. 52 (2012), 1565-1573.

[8] Abramov A.A., Yukhno L.F., A method for the numerical solution of the Painlevé equations, Comput. Math. Math. Phys. 53 (2013), 540-563.

[9] Abramov A.A., Yukhno L.F., Numerical solution of the Painlevé V equation, Comput. Math. Math. Phys. 53 (2013), 44-56.

[10] Abramov A.A., Yukhno L.F., Numerical solution of the Painlevé VI equation, Comput. Math. Math. Phys. 53 (2013), 180-193.

[11] Abramov A.A., Yukhno L.F., A method for calculating the Painlevé transcendents, Appl. Numer. Math. 93 (2015), 262-269.

[12] Babich M.V., Bordag L.A., Projective differential geometrical structure of the Painlevé equations, J. Differential Equations 157 (1999), 452-485.

[13] Bagderina Yu.Yu., Equivalence of the ordinary differential equations $y^{\prime \prime}=R(x, y) y^{\prime 2}+2 Q(x, y) y^{\prime}+P(x, y)$, Differ. Equ. 43 (2007), 595-604.

[14] Bagderina Yu.Yu., Equivalence of third-order ordinary differential equations to Chazy equations I-XIII, Stud. Appl. Math. 120 (2008), 293-332.

[15] Bagderina Yu.Yu., Invariants of a family of scalar second-order ordinary differential equations, J. Phys. A: Math. Theor. 46 (2013), 295201, 36 pages.

[16] Bagderina Yu.Yu., Equivalence of second-order ODEs to equations of first Painlevé equation type, Ufa Math. J. 7 (2015), 19-30.

[17] Bagderina Yu.Yu., Equivalence of second-order ordinary differential equations to Painlevé equations, Theoret. and Math. Phys. 182 (2015), 211-230.

[18] Bagderina Yu.Yu., Invariants of a family of scalar second-order ordinary differential equations for Lie symmetries and first integrals, J. Phys. A: Math. Theor. 49 (2016), 155202, 32 pages.

[19] Bagderina Yu.Yu., Tarkhanov N.N., Solution of the equivalence problem for the third Painlevé equation, J. Math. Phys. 56 (2015), 013507, 15 pages.

[20] Barashenkov I.V., Pelinovsky D.E., Exact vortex solutions of the complex sine-Gordon theory on the plane, Phys. Lett. B 436 (1998), 117-124.

[21] Bassom A.P., Clarkson P.A., Law C.K., McLeod J.B., Application of uniform asymptotics to the second Painlevé transcendent, Arch. Rational Mech. Anal. 143 (1998), 241-271, arXiv:solv-int/9609005.

[22] Berth M., Czichowski G., Using invariants to solve the equivalence problem for ordinary differential equations, Appl. Algebra Engrg. Comm. Comput. 11 (2001), 359-376.

[23] Bertola M., On the location of poles for the Ablowitz-Segur family of solutions to the second Painlevé equation, Nonlinearity 25 (2012), 1179-1185, arXiv:1203.2988.

[24] Bertola M., Bothner T., Zeros of large degree Vorob'ev-Yablonski polynomials via a Hankel determinant identity, Int. Math. Res. Not. 2015 (2015), 9330-9399, arXiv:1401.1408.

[25] Bogatskiy A., Claeys T., Its A., Hankel determinant and orthogonal polynomials for a Gaussian weight with a discontinuity at the edge, Comm. Math. Phys. 347 (2016), 127-162, arXiv:1507.01710.

[26] Bornemann F., On the numerical evaluation of distributions in random matrix theory: a review, Markov Process. Related Fields 16 (2010), 803-866, arXiv:0904.1581.

[27] Bornemann F., On the numerical evaluation of Fredholm determinants, Math. Comp. 79 (2010), 871-915, arXiv:0804.2543.

[28] Bothner T., Transition asymptotics for the Painlevé II transcendent, Duke Math. J. 166 (2017), 205-324, arXiv:1502.03402.

[29] Bothner T., Its A., The nonlinear steepest descent approach to the singular asymptotics of the second Painlevé transcendent, Phys. D 241 (2012), 2204-2225. 
[30] Boutroux P., Recherches sur les transcendantes de M. Painlevé et l'étude asymptotique des équations différentielles du second ordre, Ann. Sci. École Norm. Sup. (3) 30 (1913), 265-375.

[31] Boutroux P., Recherches sur les transcendantes de M. Painlevé et l'étude asymptotique des équations différentielles du second ordre (suite), Ann. Sci. École Norm. Sup. (3) 31 (1914), 99-159.

[32] Buckingham R.J., Miller P.D., Large-degree asymptotics of rational Painlevé-II functions: noncritical behaviour, Nonlinearity 27 (2014), 2489-2578, arXiv:1310.2276.

[33] Buckingham R.J., Miller P.D., Large-degree asymptotics of rational Painlevé-II functions: critical behaviour, Nonlinearity 28 (2015), 1539-1596, arXiv:1406.0826.

[34] Bureau F.J., Differential equations with fixed critical points, Ann. Mat. Pura Appl. (4) 64 (1964), $229-364$.

[35] Bureau F.J., Équations différentielles du second ordre en $Y$ et du second degré en $\ddot{Y}$ dont l'intégrale générale est à points critiques fixes, Ann. Mat. Pura Appl. (4) 91 (1972), 163-281.

[36] Bureau F.J., Garcet A., Goffar J., Transformées algébriques des équations du second ordre dont l'intégrale générale est à points critiques fixes, Ann. Mat. Pura Appl. (4) 92 (1972), 177-191.

[37] Casini H., Fosco C.D., Huerta M., Entanglement and alpha entropies for a massive Dirac field in two dimensions, J. Stat. Mech. Theory Exp. 2005 (2005), P07007, 16 pages, arXiv:cond-mat/0505563.

[38] Casini H., Huerta M., Entanglement and alpha entropies for a massive scalar field in two dimensions, J. Stat. Mech. Theory Exp. 2005 (2005), P12012, 17 pages, arXiv:cond-mat/0511014.

[39] Casini H., Huerta M., Analytic results on the geometric entropy for free fields, J. Stat. Mech. Theory Exp. 2008 (2008), P01012, 9 pages, arXiv:0707.1300.

[40] Chalkley R., New contributions to the related work of Paul Appell, Lazarus Fuchs, Georg Hamel, and Paul Painlevé on nonlinear differential equations whose solutions are free of movable branch points, J. Differential Equations 68 (1987), 72-117.

[41] Chazy J., Sur les équations différentielles dont l'intégrale générale est uniforme et admet des singularités essentielles mobiles, C. R. Acad. Sci. Paris 149 (1909), 563-565.

[42] Chazy J., Sur les équations différentielles du troisième ordre et d'ordre supérieur dont l'intégrale générale a ses points critiques fixes, Acta Math. 34 (1911), 317-385.

[43] Claeys T., Kuijlaars A.B.J., Vanlessen M., Multi-critical unitary random matrix ensembles and the general Painlevé II equation, Ann. of Math. 168 (2008), 601-641, arXiv:math-ph/0508062.

[44] Clarkson P.A., Painlevé equations - nonlinear special functions, in Orthogonal Polynomials and Special Functions, Editors F. Marcellàn, W. Van Assche, Lecture Notes in Math., Vol. 1883, Springer, Berlin, 2006, 331-411.

[45] Clarkson P.A., On Airy solutions of the second Painlevé equation, Stud. Appl. Math. 137 (2016), 93-109, arXiv:1510.08326.

[46] Clarkson P.A., Kruskal M.D., New similarity reductions of the Boussinesq equation, J. Math. Phys. 30 (1989), 2201-2213.

[47] Clarkson P.A., Mansfield E.L., The second Painlevé equation, its hierarchy and associated special polynomials, Nonlinearity 16 (2003), R1-R26.

[48] Clarkson P.A., McLeod J.B., A connection formula for the second Painlevé transcendent, Arch. Rational Mech. Anal. 103 (1988), 97-138.

[49] Clerc M.G., Dávila J.D., Kowalczyk M., Smyrnelis P., Vidal-Henriquez E., Theory of light-matter interaction in nematic liquid crystals and the second Painlevé equation, Calc. Var. Partial Differential Equations 56 (2017), Art. 93, 22 pages, arXiv:1610.03044.

[50] Conte R. (Editor), The Painlevé property. One century later, CRM Series in Mathematical Physics, Springer-Verlag, New York, 1999.

[51] Conte R., Musette M., The Painlevé handbook, Springer, Dordrecht, 2008.

[52] Cosgrove C.M., All binomial-type Painlevé equations of the second order and degree three or higher, Stud. Appl. Math. 90 (1993), 119-187.

[53] Cosgrove C.M., Painlevé classification problems featuring essential singularities, Stud. Appl. Math. 98 (1997), 355-433.

[54] Cosgrove C.M., Chazy classes IX-XI of third-order differential equations, Stud. Appl. Math. 104 (2000), 171-228. 
[55] Cosgrove C.M., Higher-order Painlevé equations in the polynomial class. I. Bureau symbol P2, Stud. Appl. Math. 104 (2000), 1-65.

[56] Cosgrove C.M., Higher-order Painlevé equations in the polynomial class. II. Bureau symbol P1, Stud. Appl. Math. 116 (2006), 321-413.

[57] Cosgrove C.M., Chazy's second-degree Painlevé equations, J. Phys. A: Math. Gen. 39 (2006), 11955-11971.

[58] Cosgrove C.M., Scoufis G., Painlevé classification of a class of differential equations of the second order and second degree, Stud. Appl. Math. 88 (1993), 25-87.

[59] Dai D., Hu W., Connection formulas for the Ablowitz-Segur solutions of the inhomogeneous Painlevé II equation, Nonlinearity 30 (2017), 2982-3009, arXiv:1611.05285.

[60] Dai D., Hu W., On the quasi-Ablowitz-Segur and quasi-Hastings-McLeod solutions of the inhomogeneous Painlevé II equation, Random Matrices Theory Appl. 7 (2018), 1840004, 13 pages, arXiv:1708.09357.

[61] Deaño A., Large $z$ asymptotics for special function solutions of Painlevé II in the complex plane, SIGMA 14 (2018), 107, 19 pages, arXiv:1804.00563.

[62] Deift P., Some open problems in random matrix theory and the theory of integrable systems, in Integrable Systems and Random Matrices, Editors J. Baik, T. Kriecherbauer, L.C. Li, K.D.T.R. McLaughlin, C. Tomei, Contemp. Math., Vol. 458, Amer. Math. Soc., Providence, RI, 2008, 419-430, arXiv:0712.0849.

[63] Deift P.A., Zhou X., Asymptotics for the Painlevé II equation, Comm. Pure Appl. Math. 48 (1995), 277337.

[64] Dodd R.K., Bullough R.K., Polynomial conserved densities for the sine-Gordon equations, Proc. Roy. Soc. London Ser. A 352 (1977), 481-503.

[65] Fasondini M., Fornberg B., Weideman J.A.C., Methods for the computation of the multivalued Painlevé transcendents on their Riemann surfaces, J. Comput. Phys. 344 (2017), 36-50.

[66] Fasondini M., Fornberg B., Weideman J.A.C., A computational exploration of the McCoy-Tracy-Wu solutions of the third Painlevé equation, Phys. D 363 (2018), 18-43.

[67] Fokas A.S., Ablowitz M.J., On a unified approach to transformations and elementary solutions of Painlevé equations, J. Math. Phys. 23 (1982), 2033-2042.

[68] Fokas A.S., Its A.R., Kapaev A.A., Novokshenov V.Yu., Painlevé transcendents. The Riemann-Hilbert approach, Mathematical Surveys and Monographs, Vol. 128, Amer. Math. Soc., Providence, RI, 2006.

[69] Fornberg B., Weideman J.A.C., A numerical methodology for the Painlevé equations, J. Comput. Phys. 230 (2011), 5957-5973.

[70] Fornberg B., Weideman J.A.C., A computational exploration of the second Painlevé equation, Found. Comput. Math. 14 (2014), 985-1016.

[71] Fornberg B., Weideman J.A.C., A computational overview of the solution space of the imaginary Painlevé II equation, Phys. D 309 (2015), 108-118.

[72] Forrester P.J., Witte N.S., Painlevé II in random matrix theory and related fields, Constr. Approx. 41 (2015), 589-613, arXiv:1210.3381.

[73] Gambier B., Sur les équations différentielles du second ordre et du premier degré dont l'intégrale générale est a points critiques fixes, Acta Math. 33 (1910), 1-55.

[74] Gariel J., Marcilhacy G., Santos N.O., Parametrization of solutions of the Lewis metric by a Painlevé transcendent III, J. Math. Phys. 47 (2006), 062502, 5 pages, arXiv:gr-qc/0012004.

[75] Garnier R., Sur des équations différentielles du troisième ordre dont l'intégrale générale est uniforme et sur une classe d'équations nouvelles d'ordre supérieur dont l'intégrale générale a ses points critiques fixes, Ann. Sci. École Norm. Sup. (3) 29 (1912), 1-126.

[76] Gromak V.I., Laine I., Shimomura S., Painlevé differential equations in the complex plane, De Gruyter Studies in Mathematics, Vol. 28, Walter de Gruyter \& Co., Berlin, 2002.

[77] Hastings S.P., McLeod J.B., A boundary value problem associated with the second Painlevé transcendent and the Korteweg-de Vries equation, Arch. Rational Mech. Anal. 73 (1980), 31-51.

[78] Hietarinta J., Dryuma V., Is my ODE a Painlevé equation in disguise?, J. Nonlinear Math. Phys. 9 (2002), suppl. 1, 67-74, arXiv:nlin.SI/0105016.

[79] Hisakado M., Unitary matrix models and Painlevé III, Modern Phys. Lett. A 11 (1996), 3001-3010, arXiv:hep-th/9609214. 
[80] Huang M., Xu S.-X., Zhang L., Location of poles for the Hastings-McLeod solution to the second Painlevé equation, Constr. Approx. 43 (2016), 463-494, arXiv:1410.3338.

[81] Ince E.L., Ordinary differential equations, Dover Publications, New York, 1944.

[82] Its A.R., Kapaev A.A., Quasi-linear Stokes phenomenon for the second Painlevé transcendent, Nonlinearity 16 (2003), 363-386, arXiv:nlin.SI/0108010.

[83] Iwasaki K., Kimura H., Shimomura S., Yoshida M., From Gauss to Painlevé. A modern theory of special functions, Aspects of Mathematics, Vol. E16, Friedr. Vieweg \& Sohn, Braunschweig, 1991.

[84] Jimbo M., Miwa T., Monodromy preserving deformation of linear ordinary differential equations with rational coefficients. II, Phys. D 2 (1981), 407-448.

[85] Joshi N., Kruskal M.D., The Painlevé connection problem: an asymptotic approach. I, Stud. Appl. Math. 86 (1992), 315-376.

[86] Joshi N., Mazzocco M., Existence and uniqueness of tri-tronquée solutions of the second Painlevé hierarchy, Nonlinearity 16 (2003), 427-439, arXiv:math.CA/0212117.

[87] Kajiwara K., Noumi M., Yamada Y., Geometric aspects of Painlevé equations, J. Phys. A: Math. Theor. 50 (2017), 073001, 164 pages, arXiv:1509.08186.

[88] Kamran N., Lamb K.G., Shadwick W.F., The local equivalence problem for $d^{2} y / d x^{2}=F(x, y, d y / d x)$ and the Painlevé transcendents, J. Differential Geom. 22 (1985), 139-150.

[89] Kapaev A., Global asymptotics of the second Painlevé transcendent, Phys. Lett. A 167 (1992), 356-362.

[90] Kartak V.V., Equivalence classes of the second order ODEs with the constant Cartan invariant, J. Nonlinear Math. Phys. 18 (2011), 613-640, arXiv:1106.6124.

[91] Kartak V.V., Solution of the equivalence problem for the Painlevé IV equation, Theoret. and Math. Phys. 173 (2012), 1541-1564.

[92] Kartak V.V., Point classification of second order ODEs and its application to Painlevé equations, J. Nonlinear Math. Phys. 20 (2013), suppl. 1, 110-129, arXiv:1204.0174.

[93] Kartak V.V., "Painlevé 34" equation: equivalence test, Commun. Nonlinear Sci. Numer. Simul. 19 (2014), 2993-3000, arXiv:1302.2419.

[94] Kitaev A.V., Vartanian A.H., Connection formulae for asymptotics of solutions of the degenerate third Painlevé equation. I, Inverse Problems 20 (2004), 1165-1206, arXiv:math.CA/0312075.

[95] Kitaev A.V., Vartanian A.H., Connection formulae for asymptotics of solutions of the degenerate third Painlevé equation: II, Inverse Problems 26 (2010), 105010, 58 pages, arXiv:1005.2677.

[96] Klein C., Stoilov N., Numerical approach to Painlevé transcendents on unbounded domains, SIGMA 14 (2018), 068, 10 pages, arXiv:1807.04442.

[97] Kossovskiy I., Zaitsev D., Normal form for second order differential equations, J. Dyn. Control Syst. 24 (2018), 541-562, arXiv:1611.08532.

[98] Kruskal M.D., Clarkson P.A., The Painlevé-Kowalevski and poly-Painlevé tests for integrability, Stud. Appl. Math. 86 (1992), 87-165.

[99] Kruskal M.D., Joshi N., Halburd R., Analytic and asymptotic methods for nonlinear singularity analysis: a review and extensions of tests for the Painlevé property, in Integrability of Nonlinear Systems (Pondicherry, 1996), Editors Y. Kosmann-Schwarzbach, B. Grammaticos, K.M. Tamizhman, Lecture Notes in Phys., Vol. 495, Springer, Berlin, 1997, 171-205, arXiv:solv-int/9710023.

[100] Levi D., Sekera D., Winternitz P., Lie point symmetries and ODEs passing the Painlevé test, J. Nonlinear Math. Phys. 25 (2018), 604-617, arXiv:1712.09811.

[101] Lund F., Example of a relativistic, completely integrable, Hamiltonian system, Phys. Rev. Lett. 38 (1977), $1175-1178$.

[102] Lund F., Regge T., Unified approach to strings and vortices with soliton solutions, Phys. Rev. D 14 (1976), 1524-1535.

[103] Mikhailov A.V., Integrability of a two-dimensional generalization of the Toda chain, JETP Lett. 30 (1979), 414-418.

[104] Mikhailov A.V., The reduction problem and the inverse scattering method, Phys. D 3 (1981), $73-117$.

[105] Miller P.D., On the increasing tritronquée solutions of the Painlevé-II equation, SIGMA 14 (2018), 125, 38 pages, arXiv:1804.03173. 
[106] Milson R., Valiquette F., Point equivalence of second-order ODEs: maximal invariant classification order, J. Symbolic Comput. 67 (2015), 16-41, arXiv:1208.1014.

[107] Muğan U., Jrad F., Non-polynomial third order equations which pass the Painlevé test, Z. Naturforsch. A 59 (2004), 163-180.

[108] Nijhoff F.W., Papageorgiou V.G., Similarity reductions of integrable lattices and discrete analogues of the Painlevé II equation, Phys. Lett. A 153 (1991), 337-344.

[109] Noumi M., Painlevé equations through symmetry, Translations of Mathematical Monographs, Vol. 223, Amer. Math. Soc., Providence, RI, 2004.

[110] Novokshenov V.Yu., Padé approximations for Painlevé I and II transcendents, Theoret. and Math. Phys. 159 (2009), 853-862.

[111] Novokshenov V.Yu., Tronquée solutions of the Painlevé II equation, Theoret. and Math. Phys. 172 (2012), 1136-1146.

[112] Novokshenov V.Yu., Distributions of poles to Painlevé transcendents via Padé approximations, Constr. Approx. 39 (2014), 85-99.

[113] Ohyama Y., Kawamuko H., Sakai H., Okamoto K., Studies on the Painlevé equations. V. Third Painlevé equations of special type $P_{\mathrm{III}}\left(D_{7}\right)$ and $P_{\mathrm{III}}\left(D_{8}\right)$, J. Math. Sci. Univ. Tokyo 13 (2006), 145-204.

[114] Ohyama Y., Okumura S., A coalescent diagram of the Painlevé equations from the viewpoint of isomonodromic deformations, J. Phys. A: Math. Gen. 39 (2006), 12129-12151, arXiv:math.CA/0601614.

[115] Okamoto K., Polynomial Hamiltonians associated with Painlevé equations. I, Proc. Japan Acad. Ser. A Math. Sci. 56 (1980), 264-268.

[116] Okamoto K., Polynomial Hamiltonians associated with Painlevé equations. II. Differential equations satisfied by polynomial Hamiltonians, Proc. Japan Acad. Ser. A Math. Sci. 56 (1980), 367-371.

[117] Okamoto K., Studies on the Painlevé equations. III. Second and fourth Painlevé equations, $P_{\mathrm{II}}$ and $P_{\mathrm{IV}}$, Math. Ann. 275 (1986), 221-255.

[118] Olver F.W.J., Lozier D.W., Boisvert R.F., Clark C.W. (Editors), NIST handbook of mathematical functions, Cambridge University Press, Cambridge, 2010, Release 1.0.21 of 2018-12-15 available at http://dlmf.nist.gov.

[119] Olver S., Numerical solution of Riemann-Hilbert problems: Painlevé II, Found. Comput. Math. 11 (2011), 153-179.

[120] Olver S., A general framework for solving Riemann-Hilbert problems numerically, Numer. Math. 122 (2012), 305-340.

[121] Olver S., Trogdon T., Nonlinear steepest descent and numerical solution of Riemann-Hilbert problems, Comm. Pure Appl. Math. 67 (2014), 1353-1389, arXiv:1205.5604.

[122] Olver S., Trogdon T., Numerical solution of Riemann-Hilbert problems: random matrix theory and orthogonal polynomials, Constr. Approx. 39 (2014), 101-149, arXiv:1210.2199.

[123] Painlevé P., Sur les équations différentielles du second ordre à points critiques fixés, C. R. Acad. Sci. Paris 127 (1898), 945-948.

[124] Periwal V., Shevitz D., Unitary-matrix models as exactly solvable string theories, Phys. Rev. Lett. 64 (1990), 1326-1329.

[125] Pohlmeyer K., Integrable Hamiltonian systems and interactions through quadratic constraints, Comm. Math. Phys. 46 (1976), 207-221.

[126] Reeger J.A., Fornberg B., Painlevé IV with both parameters zero: a numerical study, Stud. Appl. Math. 130 (2013), 108-133.

[127] Reeger J.A., Fornberg B., Painlevé IV: a numerical study of the fundamental domain and beyond, Phys. D $\mathbf{2 8 0 / 2 8 1 ~ ( 2 0 1 4 ) , ~ 1 - 1 3 . ~}$

[128] Sakai H., Rational surfaces associated with affine root systems and geometry of the Painlevé equations, Comm. Math. Phys. 220 (2001), 165-229.

[129] Sakka A., Muğan U., Second-order second degree Painlevé equations related with Painlevé I, II, III equations, J. Phys. A: Math. Gen. 30 (1997), 5159-5177.

[130] Sakka A., Muğan U., Second-order second-degree Painlevé equations related to Painlevé IV, V, VI equations, J. Phys. A: Math. Gen. 31 (1998), 2471-2490. 
[131] Segur H., Ablowitz M.J., Asymptotic solutions of nonlinear evolution equations and a Painlevé transcendent, Phys. D 3 (1981), 165-184.

[132] Tracy C.A., Widom H., Random unitary matrices, permutations and Painlevé, Comm. Math. Phys. 207 (1999), 665-685, arXiv:math.CO/9811154.

[133] Trogdon T., Olver S., Riemann-Hilbert problems, their numerical solution, and the computation of nonlinear special functions, Society for Industrial and Applied Mathematics (SIAM), Philadelphia, PA, 2016.

[134] Troy W.C., The role of Painleve II in predicting new liquid crystal self-assembly mechanisms, Arch. Ration. Mech. Anal. 227 (2018), 367-385.

[135] Tzitzéica G., Sur une nouvelle classe de surfaces, C. R. Acad. Sci. Paris 144 (1907), 1257-1259.

[136] Tzitzéica G., Sur une nouvelle classe de surfaces, Rend. Circolo Mat. Palermo 25 (1908), 180-187.

[137] Tzitzéica G., Sur une nouvelle classe de surfaces, C. R. Acad. Sci. Paris 150 (1910), 955-956.

[138] Umemura H., Painlevé equations and classical functions, Sugaku Expositions 11 (1998), 77-100.

[139] Valiquette F., Solving local equivalence problems with the equivariant moving frame method, SIGMA 9 (2013), 029, 43 pages, arXiv:1304.1616.

[140] Van Assche W., Orthogonal polynomials and Painlevé equations, Australian Mathematical Society Lecture Series, Vol. 27, Cambridge University Press, Cambridge, 2018.

[141] Vorob'ev A.P., On the rational solutions of the second Painlevé equation, Differ. Equ. 1 (1965), 79-81.

[142] Wechslberger G., Bornemann F., Automatic deformation of Riemann-Hilbert problems with applications to the Painlevé II transcendents, Constr. Approx. 39 (2014), 151-171, arXiv:1206.2446.

[143] Yablonskii A.I., On rational solutions of the second Painlevé equation, Vesti AN BSSR, Ser. Fiz.-Tech. Nauk (1959), no. 3, 30-35.

[144] Yamada Y., Padé method to Painlevé equations, Funkcial. Ekvac. 52 (2009), 83-92.

[145] Yumaguzhin V.A., Differential invariants of second order ODEs. I, Acta Appl. Math. 109 (2010), 283-313, arXiv:0804.0674.

[146] Zhiber A.V., Shabat A.B., Klein-Gordon equations with a nontrivial group, Soviet Phys. Dokl. 24 (1979), 607-609. 